\title{
Multivariate Granger Causality Analysis of Acupuncture Effects in Mild Cognitive Impairment Patients: An fMRI Study
}

\author{
Shangjie Chen, ${ }^{1}$ Lijun Bai, ${ }^{2}$ Maosheng Xu, ${ }^{1}$ Fang Wang, ${ }^{1}$ Liang Yin, ${ }^{1}$ Xuming Peng, \\ Xinghua Chen, ${ }^{3}$ and Xuemin $\mathrm{Shi}^{4}$ \\ ${ }^{1}$ Baoan Hospital, Southern Medical University, Shenzhen 518101, China \\ ${ }^{2}$ The Key Laboratory of Biomedical Information Engineering of Ministry of Education, Department of Biomedical Engineering, \\ School of Life Science and Technology, Xian Jiaotong University, Xi'an 710049, China \\ ${ }^{3}$ The First Affiliated Hospital, Guangzhou University of Traditional Chinese Medicine, Guangzhou 510405, China \\ ${ }^{4}$ The First Affiliated Hospital, Tianjin University of Traditional Chinese Medicine, Tianjin 300193, China
}

Correspondence should be addressed to Lijun Bai; bailj4152615@gmail.com and Xuemin Shi; sxmtjtcm@gmail.com

Received 21 February 2013; Revised 11 April 2013; Accepted 2 June 2013

Academic Editor: Baixiao Zhao

Copyright (C) 2013 Shangjie Chen et al. This is an open access article distributed under the Creative Commons Attribution License, which permits unrestricted use, distribution, and reproduction in any medium, provided the original work is properly cited.

Evidence from clinical reports has indicated that acupuncture has a promising effect on mild cognitive impairment (MCI). However, it is still unknown that by what way acupuncture can modulate brain networks involving the MCI. In the current study, multivariate Granger causality analysis (mGCA) was adopted to compare the interregional effective connectivity of brain networks by varying needling depths (deep acupuncture, DA; superficial acupuncture, SA) and at different cognitive states, which were the MCI and healthy control (HC). Results from DA at KI3 in MCI showed that the dorsolateral prefrontal cortex and hippocampus emerged as central hubs and had significant causal influences with each other, but significant in HC for DA. Moreover, only several brain regions had remarkable causal interactions following SA in MCI and even few brain regions following SA in HC. Our results indicated that acupuncture at KI3 at different cognitive states and with varying needling depths may induce distinct reorganizations of effective connectivities of brain networks, and DA at KI3 in MCI can induce the strongest and more extensive effective connectivities related to the therapeutic effect of acupuncture in MCI. The study demonstrated the relatively functional specificity of acupuncture at KI3 in MCI, and needling depths play an important role in acupuncture treatments.

\section{Introduction}

Mild cognitive impairment (MCI) is the key and hot point in cognitive-brain study. Incidence of dementia is widely acknowledged to increase greatly with advancing age. Sousa and coworkers report that dementia made the largest contribution to disability in China, Cuba, Dominican Republic, Mexico, Peru, and urban India [1]. Dementia is a leading cause of death in the United States but is underrecognized as a terminal illness. The median survival was 478 days, and the probability of death within 6 months was $24.7 \%$ [2]. Multivariable analyses show that dementia and cognitive impairment are by far the most strongly and independently associated chronic health disorders [3]. Increasing evidence shows that subtle losses in cognitive function may be a symptomatic transition to early $\mathrm{AD}$ [4]. $\mathrm{MCI}$ is an intermediate state between normal aging and Alzheimer's disease (AD) which is the world's most common dementia $[5,6]$. MCI represents a significant risk factor for the development of dementia [4] and is an appropriate condition for investigation [4, 7]. Further research is needed on treatments of delaying the conversion from MCI to $\mathrm{AD}$ [4]. However, there is no sufficient evidence that drug can delay long-term progression and conversion to dementia $[1,6]$. Feasible complementary and alternative therapies with low side effects, such as acupuncture and exercise, have shown some benefits $[8,9]$. The use of acupuncture as a complementary therapeutic way to treat a variety of neurologic diseases, including $\mathrm{MCI}$ and $\mathrm{AD}$, is popular in certain parts of the world [10]. In spite of its public acceptance and promising effect, the underlying neural mechanism is still elusive.

Since the late 1990s, functional magnetic resonance imaging (fMRI) has been used to investigate the underlying 
mechanisms of acupuncture [11], especially the relative functional specificity of acupoints. Neuroimaging studies have indicated that the primary acupuncture effects are mediated by the central nervous system [12-20], and acupuncture can activate certain cognitive-related regions in $\mathrm{AD}$ and $\mathrm{MCI}$ patients [9]. KI3 is one of the most frequently used acupoint in treatment of cognitive impairment [21]. Our previous studies have also indicated that acupuncture at KI3 can activate certain cognitive-related regions [22-25].

However, there are several problems in fMRI studies of acupuncture. Firstly, most of these studies have been performed on healthy subjects [12-20]. However, acupoint selection often has a very wide range of therapeutic actions related to functional state of the human body based on theory of the traditional chinese medicine (TCM). It is generally accepted that acupuncture plays a homeostatic role and thus may have a greater effect on patients with a pathological imbalance compared with healthy controls (HC) [7, 26, 27]. Therefore, exploring brain function evoked by acupuncture in patients may further help to elucidate its mechanism. In addition, most fMRI studies focus on comparison of brain activity patterns induced by acute effects of acupuncture at acupoint and nonacupoint, or different acupoints. However, few studies have evaluated the modulated effects in the poststimulus resting state networks (RSN) induced by varied needling depth (DA, deep acupuncture; SA, superficial acupuncture) though the depth of needling is also the key of specificity of acupuncture according to the theory of TCM. A primary interest in this area is therefore whether these different depths of needling elicit similar or different responses. Deep acupuncture may better overlap with its proximity to ascending nerve tracks than to the density of cutaneous afferents [28]. In addition, SA has been assumed to minimize the therapeutic effect while triggering most of the nonspecific effects of needling [29]. Therefore, comparing connectivity patterns of brain regions modulated by DA with that of the effects modulated by SA may provide precise and specific modulatory patterns related to the therapeutic effect of acupuncture.

Moreover, many studies generally adopted the blockdesigned fMRI paradigm conform to the "on-off" specifications. But, function responses induced by acupuncture have time-varying characteristics [15-18]. In recent years, some studies started to pay attention to the sustained effect of the acupuncture and its influence on the postacupuncture RSN with the functional connectivity analysis, which was a kind of undirected graph analysis of temporal correlations between different brain regions [7, 14, 16]. However, little was known about the direction and strength of the information flow between these brain regions modulated by acupuncture. Further investigation of the interregional causal interactions may be helpful to explain the neurophysiological action underlying acupuncture $[20,23]$. Recently, a newly multivariate Granger causality analysis (mGCA) has been introduced as an effective connectivity method to analyze direct causal interactions among multiple brain areas from fMRI data $[20,23,30-33]$. By exploring this approach to analyze the causal influences of the activated regions evoked by acupuncture, we can account for its modulatory effects on multiple relevant regions simultaneously.
Based on the previous study, we employed the mGCA to evaluate the effective connectivity patterns among multiple brain regions following acupuncture at KI3 in MCI patients and $\mathrm{HC}$ for DA and SA condition. By examining the directionality and strength of causal influence between multiple brain regions following acupuncture at $\mathrm{KI} 3$ in MCI patients and $\mathrm{HC}$ for DA and SA condition, we can find whether there is relatively specific modulatory effect at different cognitive states (MCI and HC) and with varying different depths of needling (DA and SA). By detecting the functional specificity of acupuncture for different cognitive states and varied depths of needling during the postresting acupuncture period, we can provide further evidence to explore the relative functional specificity of acupuncture effects.

\section{Materials and Methods}

2.1. Subjects. A total of 24 subjects were recruited in the study. 12 MCI patients ( 1 males and 11 females; ages $59.3 \pm 3.3$ years; MMSE 26.4 \pm 0.9; 7 middle school education and 5 college degree) and 12 age-matched HC subjects (4 males and 8 females; ages $60.6 \pm 5.8$ years; MMSE $29.8 \pm 0.4 ; 9$ middle school education and 3 college degree) were included. All subjects were right handed with normal or corrected-tonormal vision and acupuncture naive according to the edinburgh handedness inventory [34]. MCI patients were diagnosed by using the criteria for amnestic MCI [35], with MiniMental State Examination (MMSE) scores $>25$ [36] and Clinical Dementia Rating (CDR) scale scores of 0.5 [37]. Subjects were excluded if they had serious medical, neurological, or psychiatric illness, or if they were taking medication or other substances known to influence cerebral function, or if they have any contraindications to exposure to a high magnetic field. After being fully explained of the study, all subjects signed the informed consent. All protocols were approved by a local subcommittee on human studies and were conducted in accordance with the Declaration of Helsinki.

\subsection{Experimental Procedures. For each subject, functional} runs lasting for $15 \mathrm{~min}$ of the experiment consisted of four phases (Figure 1(b)). In the first phase, a resting state (REST) scan was first conducted for 6 min without any stimulation for a baseline control. In the second phase, an acupuncture needle was then inserted in acupoint KI3 (Taixi, located in a depression between the medial malleolus and heel tendon, Figure 1(a)) on the right leg, and retained for $1 \mathrm{~min}$. In the third phase, the needle was manipulated for $2 \mathrm{~min}$ by rotating the needle at a rate of 120 times per min in frequency and at $60^{\circ}$ in angle. In the end, another REST scan was conducted for $6 \mathrm{~min}$ without any stimulation again. All participants were asked to remain relaxed without engaging in any mental tasks. To facilitate blinding, they were also instructed to keep their eyes closed during fMRI scan to prevent them from actually observing the procedures. According to participants' reports after the scanning, they were affirmed keeping awake during the whole process. Standard acupuncture needles, which are made of ferromagnetic steel, however, are problematic in fMRI studies for several reasons, such as attraction by the scanner's magnetic field, significant image distortions, 


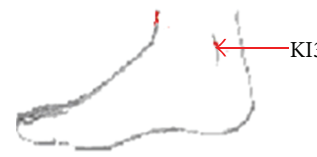

(a)

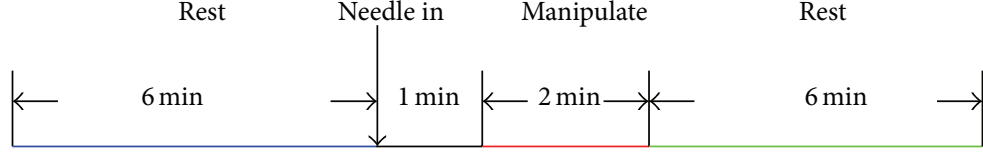

(b)

FIGURE 1: (a) Anatomical locations of the acupuncture stimulation points of KI3. (b) Experimental paradigm. Functional run lasting for 15 min consisted of four phases: 6-minute REST scanning was done before and after acupuncture, inserted in and retained for 1 min and manipulated for $2 \mathrm{~min}$.

and signal dropouts, when positioned close to the head or even heating due to absorption of radio frequency (RF). Nonferromagnetic metal needles seem to be the best choice for acupoints outside of the transmitter coil [38]. Therefore, acupuncture stimulation was delivered by a silver needle with $0.35 \mathrm{~mm}$ in diameter and $25 \mathrm{~mm}$ in length (silver content above 85\%, Acupuncture Supplies Company in Suzhou, China) in this study. The acupuncture procedure was conducted by the same experienced and licensed acupuncturist on all subjects.

We employed two functional runs (DA and SA) for each subject, but only one single stimulation period was given during each of these two runs. The needle was inserted vertically to a depth of $1-2 \mathrm{~cm}$ in DA, but of $1-2 \mathrm{~mm}$ in SA. Instead of inserting depth, other manipulation methods were all identical in the DA and SA groups. The presentation sequence of two runs was randomized and balanced throughout the population, and every participant performed only one run each week in order to eliminate potential long-lasting effect following acupuncture administration. All participants were not informed of the order in which these runs would be performed.

At the end of each acupuncture scan, the subjects were questioned about aching, pressure, soreness, heaviness, fullness, warmth, coolness, numbness, tingling, dull or sharp pain, and any other sensations they felt during the stimulation $[15,20,39]$. A 10-point visual analogue scale (VAS), which was scaled at $0=$ no sensation, $1-3=$ mild, $4-6=$ moderate, $7-8=$ strong, $9=$ severe, and $10=$ unbearable sensation, was adopted to self-rate the intensities about the deqi sensations $[15,39,40]$. To quantitatively summarize the full multivariate breadth and depth of the De-qi sensations for each subject, the VAS index was calculated $[15,20,39]$.

2.3. fMRI-Scanning Procedure. Magnetic resonance imaging data were collected from a $3 \mathrm{~T}$ MR scanner. A standard birdcage head coil was used, along with restraining foam pads to minimize head motion and to diminish scanner noise. For each subject, functional scans of acupuncture stimulation were taken after the anatomical scans. The scan covered the entire brain including the cerebellum and brainstem. Thirty axial slices were obtained using a $\mathrm{T}^{*}$-weighted single-shot, gradient-recalled echo planar imaging $(\mathrm{EPI})$ sequence $(\mathrm{TR}=$ $2000 \mathrm{~ms}, \mathrm{TE}=30 \mathrm{~ms}, \mathrm{FOV}=220 \mathrm{~mm} \times 220 \mathrm{~mm}$, matrix $=64$ $\times 64$, thickness $=4 \mathrm{~mm}$, Slice Space $=1 \mathrm{~mm}$, flip angle $=77^{\circ}$ ). High-resolution structural information on each subject was also acquired using three-dimensional (3D) MRI sequences with a voxel size of $1 \mathrm{~mm}^{3}$ for anatomical localization $(\mathrm{TR}=$ $2.1 \mathrm{~s}, \mathrm{TE}=4.6 \mathrm{~ms}, \mathrm{FOV}=230 \mathrm{~mm} \times 230 \mathrm{~mm}$, matrix $=256$ $\times 256$, slice thickness $=1 \mathrm{~mm}$, flip angle $=8^{\circ}$ ).

2.4. fMRI Data Analysis. Preprocessing was performed using the Statistical Parametric Mapping software (SPM5, http://www.fil.ion.ucl.ac.uk/spm/). Initially, the first five time points were discarded in order to avoid the instability of the initial MRI signal [40]. The image data underwent slice-timing correction and realignment for head motions using least-squares minimization. None of the subjects had head movements exceeding $1 \mathrm{~mm}$ on any axis and head rotation greater than one degree. A mean image created from the realigned volumes was co-registered with the subject's individual structural T1-weighted volume image [20]. Then, the images were normalized to the standard EPI template and re-sampled to a voxel size of $2 \mathrm{~mm} \times 2 \mathrm{~mm} \times 2 \mathrm{~mm}$ [41]. Subsequently, these data were filtered by using a bandpass filter $(0.01-0.08 \mathrm{~Hz})$ to reduce the effect of low-frequency drift and high-frequency noise [42, 43]. Finally, the images were smoothed spatially by using a $6 \mathrm{~mm}$ full-width-at-half maximum (FWHM) isotropic Gaussian kernel.

Taking into account of the sustained effects of acupuncture, the resting period before acupuncture was taken as the baseline. For each subject, the difference in the BOLD response was estimated at every voxel across the whole brain by using the general linear model (GLM) in SPM5. The obtained $t$-maps at individual levels were then entered into the "random effect" group analysis framework by the onesample $t$-test summary statistic $(P<0.005$, uncorrected). The statistical maps indicated the brain activation in response to acute effects of acupuncture, thereby functionally defining ROI. Each peak voxel with its nearest 10 neighbors was defined as a group ROI. Considering the anatomical variance across subjects, subject-specific peak voxels and subjectspecific ROIs were defined on individual $t$-maps as follows. The given group ROI was used as a mask and then, based on individual $t$-maps, and the voxel with the largest $t$-value within this mask served as the subject-specific peak voxel. ROIs were selected based on the acupuncture-stimulation results. Firstly, the time series from the poststimulus resting period of BOLD signal intensities from these selected ROIs were selected. Then, the time series were averaged across voxels within each ROI and normalized across subjects separately for each group to form a single vector per ROI [20].

In order to describe the effective connectivity during the postacupuncture resting period [30], the mGCA was 
used to detect causal interactions between brain regions by computing directed transfer function (DTF) from a multivariate autoregressive model of the time course of selected ROIs [20, 23]. Based on the principle of Granger causality, the DTF was rendered in a multivariate formulation [44]. Therefore, the DTF can effectively model the inherently multivariate nature of neuronal networks. The algorithm was coded in MATLAB7 (The MathWorks, Inc.) [20]. Effective connectivity graphs were constructed using the thickness of connecting lines and arrows to indicate the strength and direction of the causal influences. Only links that showed significant effective connectivity were presented in the network $(P<0.05)$. Graphs were visualized using Pajek software (http://vlado.fmf.uni-lj.si/pub/networks/pajek/).

\section{Results}

3.1. Psychophysical Response. The prevalence of deqi sensations was expressed as the percentage of the individuals in the group that reported the sensations (Figure 2(a)). Differences did exist with respect to the type of sensations. Both in the MCI and HC group, the soreness, numbness, fullness, warmth, and heaviness were found to be more frequent for DA than that of SA. Whether for the DA or SA, warmth and tingling were found to be more frequent in the MCI group than HC group.

The intensity of sensations was expressed as the average score \pm S.E. (Figure 2(b)). Differences did also exist with respect to the type of sensations. In both $\mathrm{MCI}$ and $\mathrm{HC}$ groups, the sensations of soreness, numbness, fullness, and warmth were found to be stronger for DA than SA. For both conditions, a statistical analysis found no significant difference between the MCI and HC groups with regard to the intensity of these sensations.

3.2. mGCA Result of Resting Brain Networks Modulated by Acupuncture. In this study, we explored the causal interactions within and among the resting brain networks modulated by acupuncture at $\mathrm{KI} 3$ in $\mathrm{MCI}$ and $\mathrm{HC}$, for $\mathrm{DA}$ and SA. The effective connectivity patterns of resting brain networks were described as directed graphs. The thickness of connecting lines and the directions of arrows indicated strength and directions of the causal influences (green line in Figure 3). Only significant effective connectivity $(P<0.05)$ was presented in the graphs.

Following acupuncture at KI3 in MCI-Deep, the mGCA result showed that the dorsolateral prefrontal cortex (DLPFC) and hippocampus (Hipp) emerged as central hubs. The DLPFC received causal inflows from most nodes in the brain network, including the Thalamus, Insula, middle temporal gyrus (MTG), and primary motor cortex (M1). The Hipp received causal inflows from the DLPFC, anterior cingulate cortex (ACC), orbitofrontal cortex (OFC), and Caudate. In addition, Insula received causal inflows from Thalamus. The precuneus (PreCN) received causal inflows from ACC and fusiform gyrus (FG). The secondary somatosensory cortex (SII) received causal inflows from Insula and DLPFC. Declive received causal inflows from Caudate, DLPFC, M1, and Uvula. There were strong causal inflows from Thalamus to
Insula and DLPFC, from Insula to SII and DLPFC, from Caudate to Declive, from OFC to Hipp, and from ACC to PreCN and Hipp. Of interests, we found that the DLPFC and Hipp were not only central hubs but also had significant causal influence on each other. The path weights of mGCA result for MCI-Deep were tabulated in Table 1 with significant connections shown in blue color.

Notably, several of these brain regions (the Hipp and DLPFC) mentioned above also have remarkably causal interactions following acupuncture at $\mathrm{KI} 3$ in HC-Deep, but they were more noncohesive than in MCI-Deep. Following acupuncture at $\mathrm{KI} 3$ in $\mathrm{HC}$-Deep, the mGCA result showed that the Hipp, OFC, DLPFC, and Uvula emerged as central hubs. There were strong causal inflows from ACC and OFC to Hipp, from DLPFC and Declive to Uvula, from MTG to OFC, from Cuneus to PreCN, from Insula to DLPFC and Putamen, from Hipp to Caudate and DLPFC, and from Thalamus to OFC and Insula. The path weights of mGCA result for HCDeep were tabulated in Table 2 with significant connections shown in blue color.

Only several of brain regions had remarkably causal interactions following acupuncture at KI3 in MCI-Shallow. Following acupuncture at KI3 in MCI-Shallow, the mGCA result showed that there were causal inflows from pMCC and Cuneus to PreCN, from MPFC to Cuneus, from Putamen to $\mathrm{FG}$, from $\mathrm{M} 1$ to primary somatosensory cortex (SI), and from Insula to Thalamus. The path weights of mGCA result for MCI-shallow were tabulated in Table 3 with significant connections shown in blue color. It is also notable that a few brain regions had remarkably causal interactions following acupuncture at KI3 in HC-Shallow.

\section{Discussion}

$\mathrm{KI} 3$ is one of the most frequently used acupoints and prove to have various efficacies in the treatment of dementia [15]. Our previous studies have indicated that acupuncture at KI3 can activate certain cognitive-related regions [22-25]. In this study, we have further investigation on effective connectivity of postacupuncture resting brain networks at KI3 in different cognitive states and with varying acupuncture depths.

Previous functional connectivity analysis primarily focused on the correlation patterns, and this method was limited to assess brain regions functionally connected to the initially selected seed and was unable to directly characterize interactions between multiple brain regions [20]. Few studies paid attention to the direction and strength of the information flow between these brain regions modulated by acupuncture. Effective connectivity in the poststimulus resting brain may underlie the neural mechanism of acupuncture for the treatment of MCI, but very few studies have yet investigated it. By visualizing the effective connectivity, we can obtain both the direction and strength of the information flow between multiple brain regions in the resting state network following acupuncture. In this study, a newly mGCA was employed to explore the specific effective connectivity poststimulus resting period following acupuncture at KI3. Our results demonstrated that acupuncture at KI3 in different cognitive states and with varying acupuncture depths 


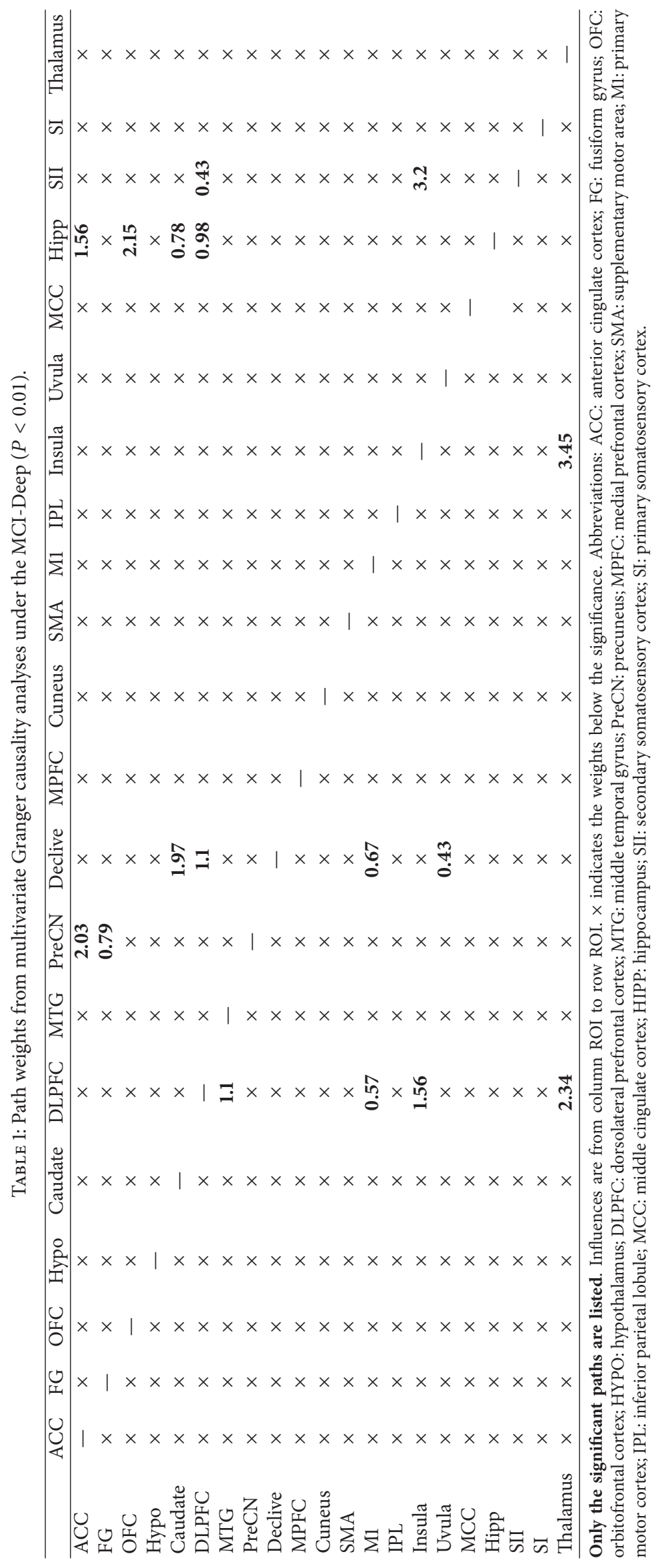




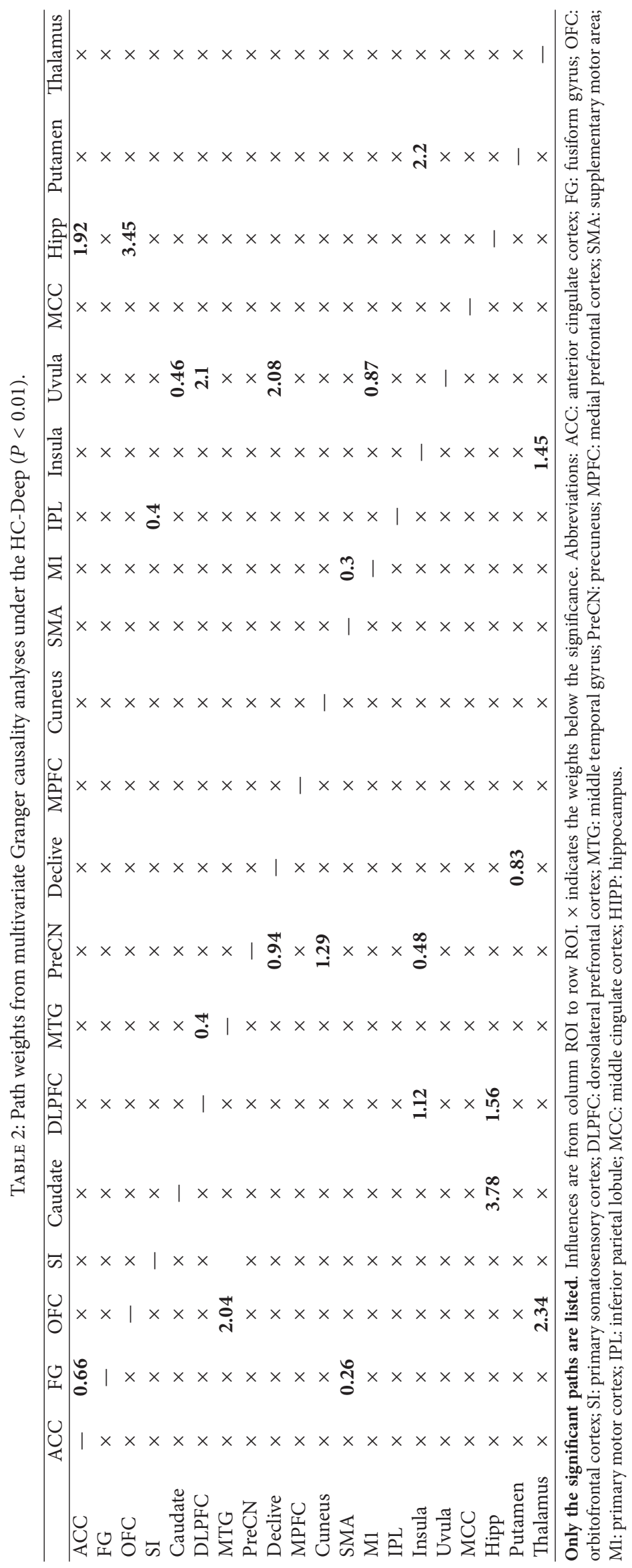



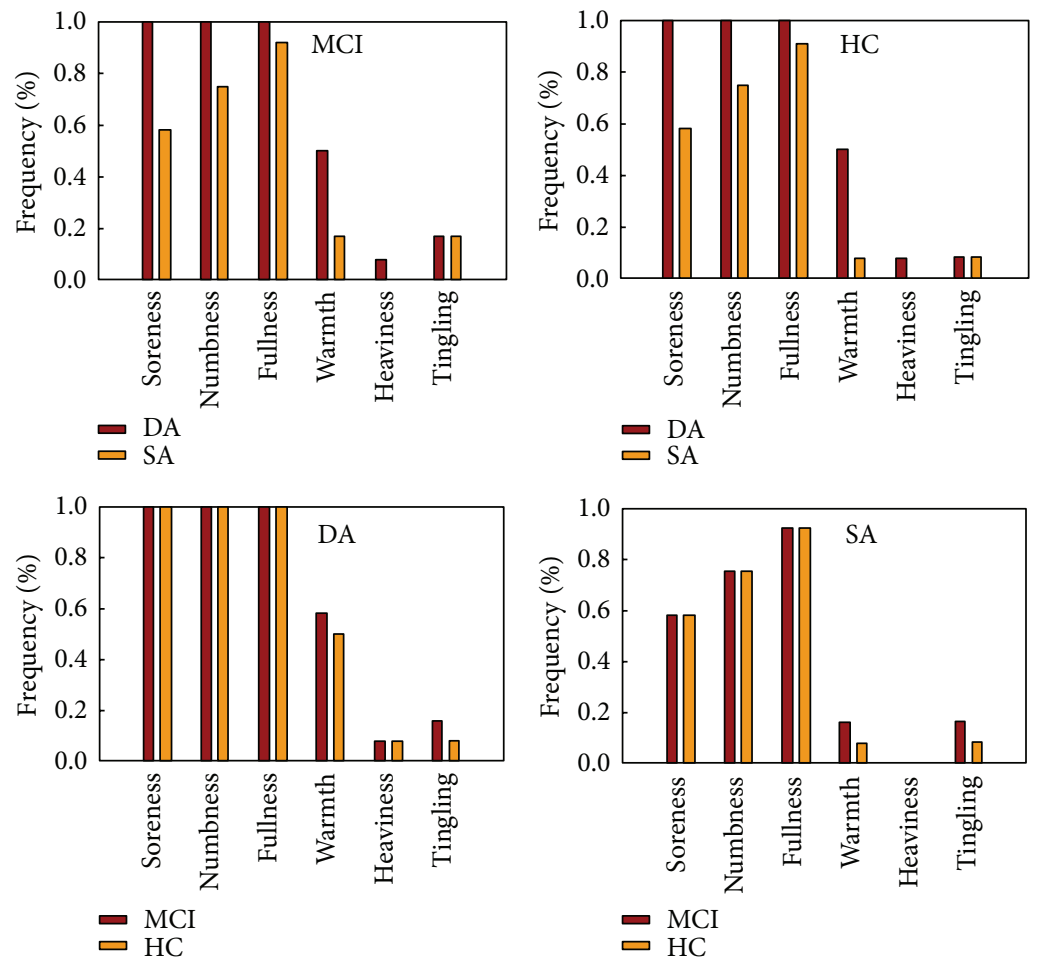

(a)
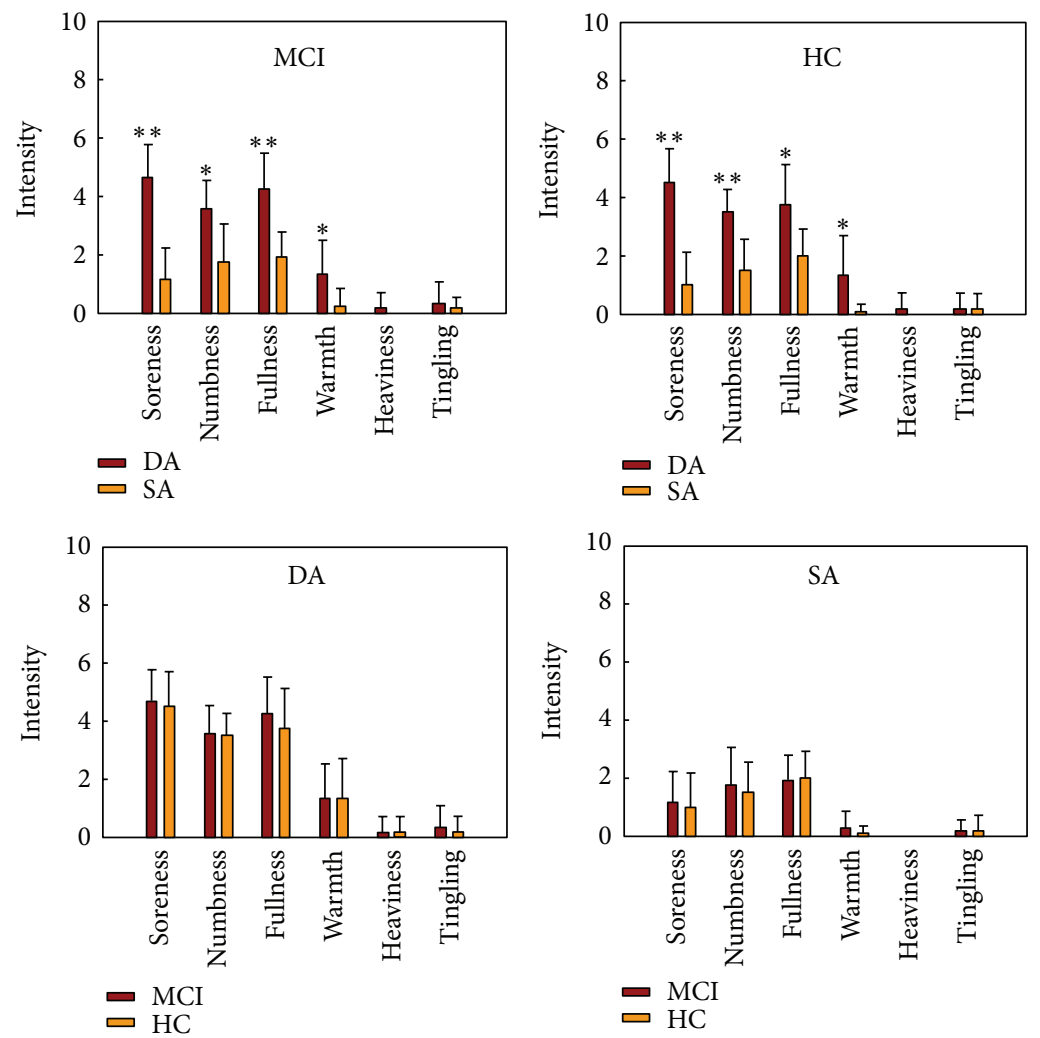

(b)

FIGURE 2: (a) The prevalence of deqi sensations. It was expressed as the percentage of the individuals in the group that reported the sensation (at least one subject experienced the seven sensations listed). (b) The intensity of sensations. It was expressed as the average score \pm S.E by measuring on a scale from 0 denoting no sensation to 10 denoting an unbearable sensation. The intensity of numbness, fullness, and soreness was found to be greater for the DA than the SA under Fisher's Exact Test $\left({ }^{*} P<0.01\right.$; $\left.{ }^{* *} P<0.001\right)$. 
TABLE 3: Path weights from multivariate Granger causality analyses under the MCI-Shallow $(P<0.01)$.

\begin{tabular}{lccccccccccccccc}
\hline & pMCC & FG & MPFC & Putamen & STG & PreCN & Culmen & Cuneus & M1 & IPL & Insula & aMCC & SI & Thalamus \\
\hline pMCC & - & $\times$ & $\times$ & $\times$ & $\times$ & $\mathbf{0 . 9 8}$ & $\times$ & $\times$ & $\times$ & $\times$ & $\times$ & $\times$ & $\times$ & $\times$ & $\times$ \\
FG & $\times$ & - & $\times$ & $\times$ & $\times$ & $\times$ & $\times$ & $\times$ & $\times$ & $\times$ & $\times$ & $\times$ & $\times$ & $\times$ & $\times$ \\
MPFC & $\times$ & $\times$ & - & $\times$ & $\times$ & $\times$ & $\times$ & $\mathbf{0 . 6 3}$ & $\times$ & $\times$ & $\times$ & $\times$ & $\times$ & $\times$ & $\times$ \\
Putamen & $\times$ & $\mathbf{0 . 7 8}$ & $\times$ & - & $\times$ & $\times$ & $\times$ & $\times$ & $\times$ & $\times$ & $\times$ & $\times$ & $\times$ & $\times$ & $\times$ \\
STG & $\times$ & $\times$ & $\times$ & $\times$ & - & $\times$ & $\times$ & $\times$ & $\times$ & $\times$ & $\times$ & $\times$ & $\times$ & $\times$ \\
PreCN & $\times$ & $\times$ & $\times$ & $\times$ & $\times$ & - & $\times$ & $\times$ & $\times$ & $\times$ & $\times$ & $\times$ & $\times$ & $\times$ & $\times$ \\
Culmen & $\times$ & $\times$ & $\times$ & $\times$ & $\times$ & $\times$ & - & & $\times$ & $\times$ & $\times$ & $\times$ & $\times$ & $\times$ \\
Cuneus & $\times$ & $\times$ & $\times$ & $\times$ & $\times$ & $\mathbf{0 . 5 6}$ & $\times$ & - & $\times$ & $\times$ & $\times$ & $\times$ & $\times$ & $\times$ \\
M1 & $\times$ & $\times$ & $\times$ & $\times$ & $\times$ & $\times$ & $\times$ & $\times$ & - & $\times$ & $\times$ & $\times$ & $\mathbf{1 . 3 4}$ & $\times$ \\
IPL & $\times$ & $\times$ & $\times$ & $\times$ & $\times$ & $\times$ & $\times$ & $\times$ & $\times$ & - & $\times$ & $\times$ & $\times$ & $\times$ \\
Insula & $\times$ & $\times$ & $\times$ & $\times$ & $\times$ & $\times$ & $\times$ & $\times$ & $\times$ & $\times$ & - & $\times$ & $\times$ & $\mathbf{0 . 3 2}$ \\
aMCC & $\times$ & $\times$ & $\times$ & $\times$ & $\times$ & $\times$ & $\times$ & $\times$ & $\times$ & $\times$ & $\times$ & - & $\times$ \\
SI & $\times$ & $\times$ & $\times$ & $\times$ & $\times$ & $\times$ & $\times$ & $\times$ & $\times$ & $\times$ & $\times$ & $\times$ & - & $\times$ \\
Thalamus & $\times$ & $\times$ & $\times$ & $\times$ & $\times$ & $\times$ & $\times$ & $\times$ & $\times$ & $\times$ & $\times$ & $\times$ & $\times$ \\
\hline
\end{tabular}

Only the significant paths are listed. Influences are from column ROI to row ROI. $\times$ indicates the weights below the significance. Abbreviations: pMCC: posterior middle cingulate cortex; FG: fusiform gyrus; MPFC: medial prefrontal cortex; STG: superior temporal gyrus; PreCN: precuneus; M1: primary motor cortex; IPL: inferior parietal lobule; aMCC: anterior middle cingulate cortex; SI: primary somatosensory cortex.

may exert heterogeneous modulatory effects on the causal interactions of brain areas during the poststimulus resting state. These different effective connectivity patterns may be related to the special effects of acupuncture in clinical settings $[20,23]$. Our findings may be helpful to understand the basic neurophysiological mechanisms underlying the specificity of acupuncture.

According to the mGCA results following acupuncture at $\mathrm{KI} 3$ in MCI-Deep, we identified that brain regions have extensive causal interactions, mainly locating at the DLPFC, Hipp, Thalamus, Insula, Declive, MTG, ACC, OFC, and Caudate. The results from mGCA showed that the DLPFC and Hipp emerged as central hubs and had significant causal influence on each other. The DLPFC received causal inflows from most nodes in the brain network, including the Thalamus, Insula, MTG, and M1. One study showed that the DLPFC disconnections may be the substrates of cognitive impairments in MCI patients [45]. The DLPFC plays a role in sustaining attention and working memory [46, 47]. Lesions in the DLPFC can impair the short-term memory and cause difficulties in inhibiting responses [46]. In addition, the DLPFC has recently been found to be involved in exhibiting self-control [47]. The inhibition of the right DLPFC could modulate the excitability of a network of brain regions, in the ipsilateral as well as in the contralateral hemisphere, to enhance function in $\mathrm{HC}$ or restore an adaptive equilibrium in the MCI [48]. In addition, the Hipp received causal inflows from the DLPFC, ACC, OFC, and Caudate. In addition, the Insula received strong causal inflows from the Thalamus. One study showed abnormalities in the connectivity associated with the hippocampus in MCI [49]. Functional results indicated that the hippocampus reduced cortical activation in the DMN for MCI patients, compared with age- and educationmatched healthy elderly controls [50]. The hippocampus plays a key role in a distributed network supporting memory encoding and retrieval [51]. The meta-analyses of 1,768 functional neuroimaging experiments revealed four functionally distinct regions on the human insula, which map to the social-emotional, the sensorimotor, the olfactory-gustatory, and the cognitive network of the brain [52]. Abnormal insula functional network is associated with episodic memory decline in amnestic mild cognitive impairment [53]. The thalamus is functionally connected to the hippocampus as part of the extended hippocampal system [54]. The literature seems to support the hypothesis that specialization of cortical areas in the MTL, as for their involvement in recollection and familiarity processes, may also extend to discrete regions of the thalamus [55]. Functional connectivity between the left thalamus and a set of regions was decreased in MCI, increased functional connectivity between the left thalamus and the right thalamus in MCI [56]. The DLPFC connectivity with the IPL and thalamus significantly correlated with the cognitive performance of patients measured by minimental state examination, clock drawing test, and California verbal learning test scores [57]. Therefore, the causal interactions related with these cognitive-related regions following DA may relate to the therapeutic effect of acupuncture for the treatment of MCI.

In addition, we also found that the hippocampus and DLPFC related with cognitive-related regions mentioned above also have remarkably causal interactions following acupuncture at KI3 in HC-Deep. Compared with that in MCI, causal interactions were significant noncohesive in HC. Many studies showed that there were specific functional changes of brain in the MCI patients compared with age- and educationmatched healthy elderly controls $[45,50]$. Acupuncture plays a homeostatic role and thus may have a greater effect on patients with a pathological imbalance compared to HC [7, 26, 27]. For HC, effect of acupuncture is weak. Our results demonstrated that there were different effective connectivities of postacupuncture at KI3 in different cognitive states, 


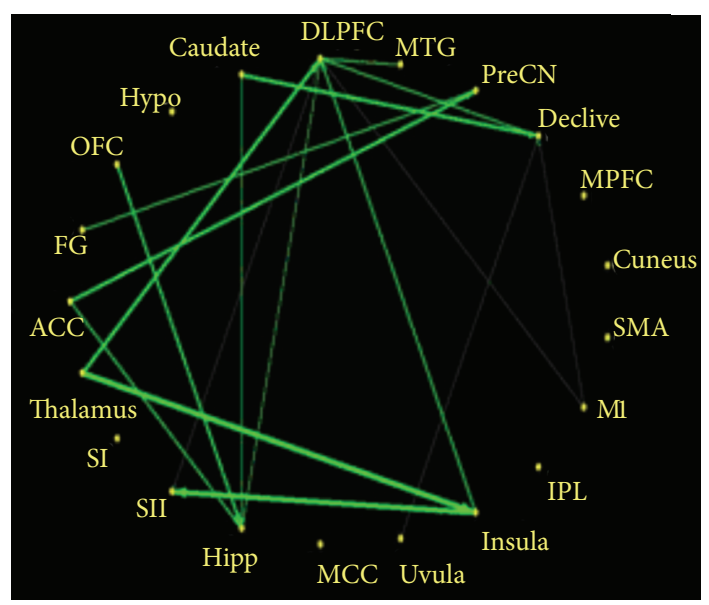

(a) MCI-Deep

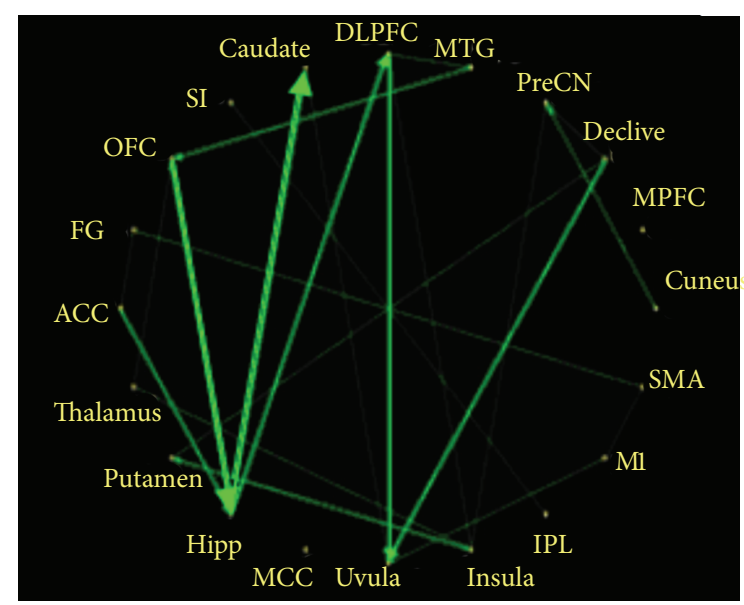

(b) HC-Deep

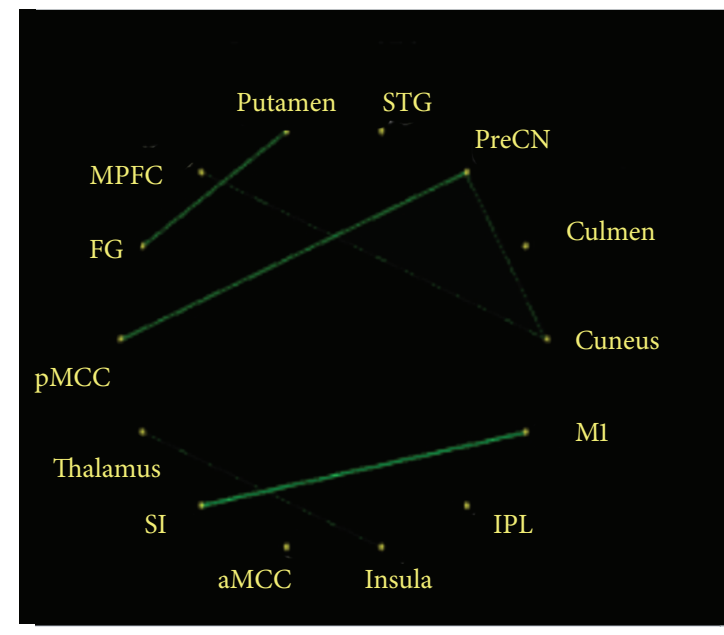

(c) MCI-Shallow

FIGURE 3: Multivariate Granger causality relationships. There were remarkably causal interactions $(P<0.05)$ following acupuncture at KI3 in MCI-Deep, HC-Deep, and MCI-Shallow, but no causal interactions following acupuncture at KI3 in HC-Shallow. Relative strength of path weights (in arbitrary units) was indicated by the width of the arrows. Abbreviations: ACC, anterior cingulate cortex; FG, fusiform gyrus; OFC, orbitofrontal cortex; HYPO, hypothalamus; DLPFC, dorsolateral prefrontal cortex; MTG, middle temporal gyrus; PreCN, precuneus; MPFC, medial prefrontal cortex; SMA, supplementary motor area; M1, primary motor cortex; IPL, inferior parietal lobule; MCC, middle cingulate cortex; HIPP, hippocampus; SII, secondary somatosensory cortex; SI, primary somatosensory cortex; pMCC, middle part of the posterior cingulate cortex; STG, superior temporal gyrus; aMCC, middle part of the anterior cingulate cortex.

and effect of acupuncture was stronger in MCI compared with that in HC.

Moreover, only several of brain regions had remarkably causal interactions following acupuncture at MCI-Shallow. Most interestingly, no brain regions had remarkably causal interactions following acupuncture at HC-Shallow. The mGCA results demonstrated that effective connectivity of postacupuncture at KI3 in shallow of needling was very weak. Furthermore, the effect of postacupuncture at KI3 in HCShallow of needling was too weak to evoke causal interactions. Deep insertion of the needle affects several structures including the skin, muscle fascia, and muscle, and acupoints may better overlap with their proximity to ascending nerve tracks than to the density of cutaneous afferents [28]. The muscular afferents affect greater number of receptors to achieve a special clinical effect than the cutaneous afferents from shallow insertion of the needle. Therefore, the stronger effective connectivity associated with the cognitive-related regions following DA may suggest that deep muscle acupuncture has a better therapeutic effect for the treatment of MCI. The heterogeneous effective connectivity patterns between DA and SA may suggest the importance of the muscular afferents in acupuncture. The results of psychophysical response showed that there were different prevalence and intensity of sensations for both conditions. Soreness, numbness, fullness, and warmth were found to be more frequent and stronger for DA than SA. Effect of acupuncture is better if sensation is stronger based on TCM. But, one study suggested that acupuncture needle stimulation at two different depths of needling, superficial, and deep did not elicit significantly different BOLD responses [58]. This result may be related to the mistaken design of block design and acupuncture in $\mathrm{HC}$. 
Moreover, the author also pointed out that the participants in that study were healthy individuals, and it is possible that superficial and deep acupuncture could potentially have different effects when being used to treat people with pathology. Our results demonstrated that there was different effective connectivity of postacupuncture at KI3 in different depths of needling, and effect of acupuncture was stronger for DA compared with that for SA.

In conclusion, results indicated that acupuncture at acupoint $\mathrm{KI} 3$ in different cognitive state and different acupuncture depth may induce distinct reorganization of the effective connectivity across different neural subsystems, and acupuncture at KI3 in MCI-Deep can induce the strongest and more extensive effective connectivity related to the therapeutic effect of acupuncture for the treatment of MCI.

\section{Conclusions}

The current study demonstrated that the significantly enhanced correlations in the cognitive-related brain regions following acupuncture may be related to the therapeutic effects of acupuncture for the treatment of MCI. Our results also revealed that there existed more tightly effective connectivity patterns during the poststimulus resting state following acupuncture at acupoint $\mathrm{KI} 3$ in MCI patients compared to $\mathrm{HC}$, stronger effective connectivity patterns for DA compared to SA, and acupuncture effects could last for a long period even though the needling process was terminated. We suggested that the distinct modulation patterns of the resting brain networks attributed to the specific effects which was evoked by acupuncture in different cognitive states and different needling depths. The study demonstrated that acupoint can play a better role in the suitable depth of needle and disease state. This preliminary finding may provide a new clue to decipher the relatively functional specificity of acupuncture effects. Our findings may help to understand the neurophysiological mechanism underlying acupuncture specificity and to employ KI3 for the treatment of MCI in the clinical practice.

\section{Conflict of Interests}

The authors declare no conflict of interests.

\section{Acknowledgments}

This study was supported by the National Natural Science Foundation of China under Grant nos. 81173354 and 81071217, the Fundamental Research Funds for the Central University, the Beijing Nova program (Grant no. Z111101054511116), the Beijing Natural Science Foundation (Grant no. 4122082), the National Key Basic Research and Development Program (973) under Grant no. 2010CB530506, the Natural Science Foundation of Guangdong Province under Grant nos. 10451810101005862 and 8451040701000553, and the Administration of Traditional Chinese Medicine of Guangdong Province under Grant no. 20111032.

\section{References}

[1] S. R. Sabat, "Dementia in developing countries: a tidal wave on the horizon," The Lancet, vol. 374, no. 9704, pp. 1805-1806, 2009.

[2] A. Hsu and H. Kao, "The clinical course of advanced dementia," New England Journal of Medicine, vol. 362, no. 4, pp. 363-365, 2010.

[3] R. M. Sousa, C. P. Ferri, D. Acosta et al., "Contribution of chronic diseases to disability in elderly people in countries with low and middle incomes: a 10/66 Dementia Research Group population-based survey," The Lancet, vol. 374, no. 9704, pp. 1821-1830, 2009.

[4] A. Levey, J. Lah, F. Goldstein, K. Steenland, and D. Bliwise, "Mild cognitive impairment: an opportunity to identify patients at high risk for progression to Alzheimer's disease," Clinical Therapeutics, vol. 28, no. 7, pp. 991-1001, 2006.

[5] R. C. Petersen, G. E. Smith, S. C. Waring, R. J. Ivnik, E. G. Tangalos, and E. Kokmen, "Mild cognitive impairment: clinical characterization and outcome," Archives of Neurology, vol. 56, no. 3, pp. 303-308, 1999.

[6] M. R. Farlow, "Treatment of mild cognitive impairment (MCI)," Current Alzheimer Research, vol. 6, no. 4, pp. 362-367, 2009.

[7] Y. Feng, L. Bai, Y. Ren et al., "FMRI connectivity analysis of acupuncture effects on the whole brain network in mild cognitive impairment patients," Magnetic Resonance Imaging, vol. 30, no. 5, pp. 672-682, 2012.

[8] H. Cheng, J. Yu, Z. Jiang et al., "Acupuncture improves cognitive deficits and regulates the brain cell proliferation of SAMP8 mice," Neuroscience Letters, vol. 432, no. 2, pp. 111-116, 2008.

[9] Z. Wang, B. Nie, D. Li et al., "Effect of acupuncture in mild cognitive impairment and Alzheimer disease: a functional MRI study," PLoS One, vol. 7, no. 8, Article ID e42730, 2012.

[10] NIH, "NIH consensus conference statement acupuncture," Journal of the American Medical Association, vol. 280, no. 17, pp. 1518-1524, 1998.

[11] F. Beissner and C. Henke, "Methodological problems in fMRI studies on acupuncture: a critical review with special emphasis on visual and auditory cortex activations," Evidence-Based Complementary and Alternative Medicine, vol. 2011, Article ID 607637, 7 pages, 2011.

[12] K. K. S. Hui, J. Liu, N. Makris et al., "Acupuncture modulates the limbic system and subcortical gray structures of the human brain: evidence from fMRI studies in normal subjects," Human Brain Mapping, vol. 9, no. 1, pp. 13-25, 2000.

[13] S. Yoo, E. Teh, R. A. Blinder, and F. A. Jolesz, "Modulation of cerebellar activities by acupuncture stimulation: evidence from fMRI study," NeuroImage, vol. 22, no. 2, pp. 932-940, 2004.

[14] R. P. Dhond, C. Yeh, K. Park, N. Kettner, and V. Napadow, "Acupuncture modulates resting state connectivity in default and sensorimotor brain networks," Pain, vol. 136, no. 3, pp. 407418, 2008.

[15] L. Bai, W. Qin, J. Tian et al., "Time-varied characteristics of acupuncture effects in fMRI studies," Human Brain Mapping, vol. 30, no. 11, pp. 3445-3460, 2009.

[16] L. Bai, W. Qin, J. Tian et al., "Acupuncture modulates spontaneous activities in the anticorrelated resting brain networks," Brain Research, vol. 1279, pp. 37-49, 2009.

[17] J. Fang, Z. Jin, Y. Wang et al., "The salient characteristics of the central effects of acupuncture needling: limbic-paralimbicneocortical network modulation," Human Brain Mapping, vol. 30, no. 4, pp. 1196-1206, 2009. 
[18] L. Bai, H. Yan, N. Li et al., "Neural specificity of acupuncture stimulation at pericardium 6: evidence from an fMRI study," Journal of Magnetic Resonance Imaging, vol. 31, no. 1, pp. 71-77, 2010.

[19] L. Bai, J. Tian, C. Zhong et al., "Acupuncture modulates temporal neural responses in wide brain networks: evidence from fMRI study," Molecular Pain, vol. 6, p. 73, 2010.

[20] Y. Feng, L. Bai, W. Zhang et al., "Investigation of acupoint specificity by multivariate granger causality analysis from functional MRI data," Journal of Magnetic Resonance Imaging, vol. 34, no. 1, pp. 31-42, 2011.

[21] Y. Zhou and J. Jia, "Effect of acupuncture given at the HT 7, ST 36, ST 40 and KI 3 acupoints on various parts of the brains of Alzheimer's disease patients," Acupuncture and Electro-Therapeutics Research, vol. 33, no. 1-2, pp. 9-17, 2008.

[22] W. Q. Qiu, J. Claunch, J. Kong et al., "The effects of acupuncture on the brain networks for emotion and cognition: an observation of gender differences," Brain Research, vol. 1362, pp. 56-67, 2010.

[23] C. Zhong, L. Bai, R. Dai et al., "Modulatory effects of acupuncture on resting-state networks: a functional MRI study combining independent component analysis and multivariate granger causality analysis," Journal of Magnetic Resonance Imaging, vol. 35, no. 3, pp. 572-581, 2012.

[24] T. Xue, L. Bai, S. Chen et al., "Neural specificity of acupuncture stimulation from support vector machine classification analysis," Magnetic Resonance Imaging, vol. 29, no. 7, pp. 943-950, 2011.

[25] S. Chen, L. Meng, H. Yan et al., "Functional organization of complex brain networks modulated by acupuncture at different acupoints belonging to the same anatomic segment," Chinese Medical Journal, vol. 125, no. 15, pp. 2694-2700, 2012.

[26] J. P. Plummer, "Acupuncture and homeostasis: physiological, physical (postural) and psychological," American Journal of Chinese Medicine, vol. 9, no. 1, pp. 1-14, 1981.

[27] T. Kaptchuk, "Acupuncture: theory, efficacy, and practice," Annals of Internal Medicine, vol. 136, no. 5, pp. 374-383, 2002.

[28] N. Goldman, M. Chen, T. Fujita et al., "Adenosine A1 receptors mediate local anti-nociceptive effects of acupuncture," Nature Neuroscience, vol. 13, no. 7, pp. 883-888, 2010.

[29] C. Vincent and G. Lewith, "Placebo controls for acupuncture studies," Journal of the Royal Society of Medicine, vol. 88, no. 4, pp. 199-202, 1995.

[30] Z. Liu, Y. Zhang, L. Bai et al., "Investigation of the effective connectivity of resting state networks in Alzheimer's disease: a functional MRI study combining independent components analysis and multivariate Granger causality analysis," NMR in Biomedicine, vol. 25, no. 12, pp. 1311-1320, 2012.

[31] G. Deshpande, S. LaConte, G. A. James, S. Peltier, and X. Hu, "Multivariate granger causality analysis of fMRI data," Human Brain Mapping, vol. 30, no. 4, pp. 1361-1373, 2009.

[32] W. Liao, J. Ding, D. Marinazzo et al., "Small-world directed networks in the human brain: multivariate Granger causality analysis of resting-state fMRI," NeuroImage, vol. 54, no. 4, pp. 2683-2694, 2011.

[33] F. Krueger, S. Landgraf, E. Van Der Meer, G. Deshpande, and $\mathrm{X}$. Hu, "Effective connectivity of the multiplication network: a functional MRI and multivariate granger causality mapping study," Human Brain Mapping, vol. 32, no. 9, pp. 1419-1431, 2011.

[34] R. Oldfield, "The assessment and analysis of handedness: the Edinburgh inventory," Neuropsychologia, vol. 9, no. 1, pp. 97-113, 1971.
[35] R. Petersen, R. Doody, A. Kurz et al., "Current concepts in mild cognitive impairment," Archives of Neurology, vol. 58, no. 12, pp. 1985-1992, 2001.

[36] S. D. Forman, J. D. Cohen, M. Fitzgerald, W. F. Eddy, M. A. Mintun, and D. C. Noll, "Improved assessment of significant activation in functional magnetic resonance imaging (fMRI): use of a cluster-size threshold," Magnetic Resonance in Medicine, vol. 33, no. 5, pp. 636-647, 1995.

[37] J. Morris, "The clinical dementia rating (CDR): current version and scoring rules," Neurology, vol. 43, no. 11, pp. 2412-2414, 1993.

[38] F. Beissner, U. Nöth, and T. Schockert, "The problem of metal needles in acupuncture-fMRI studies," Evidence-based Complementary and Alternative Medicine, vol. 2011, Article ID 808203, 5 pages, 2011.

[39] J. Kong, R. Gollub, T. Huang et al., "Acupuncture De Qi, from qualitative history to quantitative measurement," Journal of Alternative and Complementary Medicine, vol. 13, no. 10, pp. 1059-1070, 2007.

[40] J. Sun, W. Qin, L. Jin et al., "Impact of global normalization in fMRI acupuncture studies," Evidence-Based Complementary and Alternative Medicine, vol. 2012, Article ID 467061, 22 pages, 2012.

[41] O. Demirci, M. C. Stevens, N. C. Andreasen et al., "Investigation of relationships between fMRI brain networks in the spectral domain using ICA and Granger causality reveals distinct differences between schizophrenia patients and healthy controls," Neurolmage, vol. 46, no. 2, pp. 419-431, 2009.

[42] M. D. Greicius, B. Krasnow, A. L. Reiss, and V. Menon, "Functional connectivity in the resting brain: a network analysis of the default mode hypothesis," Proceedings of the National Academy of Sciences of the United States of America, vol. 100, no. 1, pp. 253-258, 2003.

[43] Q. Jiao, G. Lu, Z. Zhang et al., "Granger causal influence predicts BOLD activity levels in the default mode network," Human Brain Mapping, vol. 32, no. 1, pp. 154-161, 2011.

[44] K. J. Blinowska, R. Kuś, and M. Kamiński, "Granger causality and information flow in multivariate processes," Physical Review E, vol. 70, no. 5, Article ID 050902, pp. 50902-50606, 2004.

[45] P. Liang, Z. Wang, Y. Yang, X. Jia, and K. Li, "Functional disconnection and compensation in mild cognitive impairment: evidence from DLPFC connectivity using resting-state fMRI," PLoS ONE, vol. 6, no. 7, Article ID e22153, 2011.

[46] M. Petrides, "Dorsolateral prefrontal cortex: comparative cytoarchitectonic analysis in the human and the macaque brain and corticocortical connection patterns," European Journal of Neuroscience, vol. 11, no. 3, pp. 1011-1036, 1999.

[47] T. A. Hare, C. F. Camerer, and A. Rangel, "Self-control in decision-making involves modulation of the vmPFC valuation system," Science, vol. 324, no. 5927, pp. 646-648, 2009.

[48] P. Turriziani, D. Smirni, G. Zappalà, G. R. Mangano, M. Oliveri, and L. Cipolotti, "Enhancing memory performance with rTMS in healthy subjects and individuals with mild cognitive impairment: the role of the right dorsolateral prefrontal cortex," Frontiers in Human Neuroscience, vol. 6, p. 62, 2012.

[49] F. Bai, Z. Zhang, D. R. Watson et al., "Abnormal functional connectivity of hippocampus during episodic memory retrieval processing network in amnestic mild cognitive impairment," Biological Psychiatry, vol. 65, no. 11, pp. 951-958, 2009.

[50] F. D. Vogelaere, P. Santens, E. Achten, P. Boon, and G. Vingerhoets, "Altered default-mode network activation in mild cognitive impairment compared with healthy aging," Neuroradiology, vol. 54, no. 11, pp. 1195-1206, 2012. 
[51] M. Moser and E. I. Moser, "Distributed encoding and retrieval of spatial memory in the hippocampus," Journal of Neuroscience, vol. 18, no. 18, pp. 7535-7542, 1998.

[52] F. Kurth, K. Zilles, P. T. Fox, A. R. Laird, and S. B. Eickhoff, "A link between the systems: functional differentiation and integration within the human insula revealed by meta-analysis," Brain structure \& Function, vol. 214, no. 5-6, pp. 519-534, 2010.

[53] C. Xie, F. Bai, H. Yu et al., "Abnormal insula functional network is associated with episodic memory decline in amnestic mild cognitive impairment," Neuroimage, vol. 63, no. 1, pp. 320-327, 2012.

[54] T. Stein, C. Moritz, M. Quigley, D. Cordes, V. Haughton, and E. Meyerand, "Functional connectivity in the thalamus and hippocampus studied with functional MR imaging," American Journal of Neuroradiology, vol. 21, no. 8, pp. 1397-1401, 2000.

[55] G. A. Carlesimo, M. G. Lombardi, and C. Caltagirone, "Vascular thalamic amnesia: a reappraisal," Neuropsychologia, vol. 49, no. 5, pp. 777-789, 2011.

[56] Z. Wang, X. Jia, P. Liang et al., "Changes in thalamus connectivity in mild cognitive impairment: evidence from resting state fMRI," European Journal of Radiology, vol. 81, no. 2, pp. 277-285, 2012.

[57] P. Liang, Z. Wang, Y. Yang, X. Jia, and K. Li, "Functional disconnection and compensation in mild cognitive impairment: evidence from DLPFC connectivity using resting-state fMRI," PLoS ONE, vol. 6, no. 7, Article ID e22153, 2011.

[58] H. MacPherson, G. Green, A. Nevado et al., "Brain imaging of acupuncture: comparing superficial with deep needling," Neuroscience Letters, vol. 434, no. 1, pp. 144-149, 2008. 


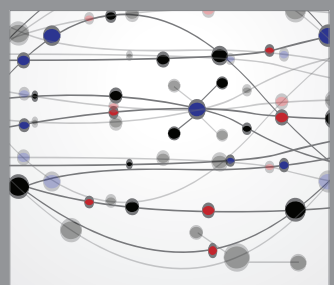

The Scientific World Journal
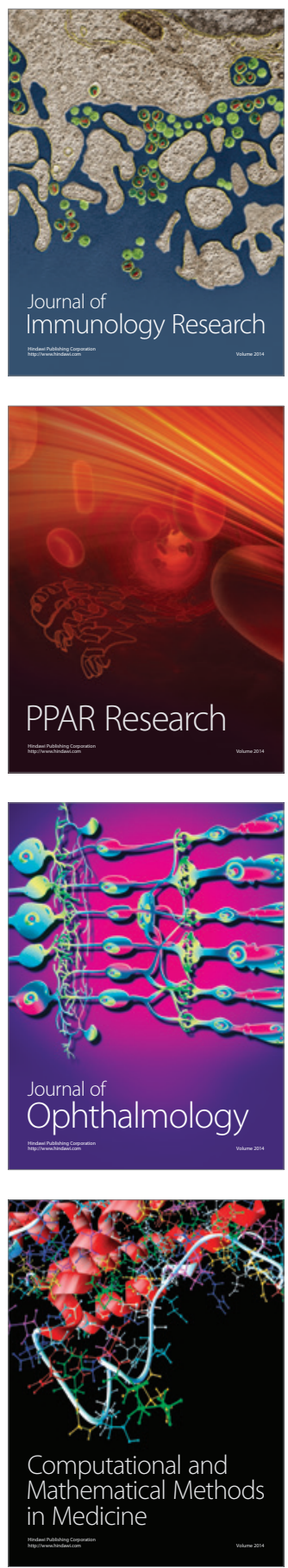

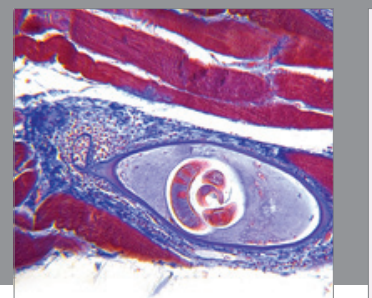

Gastroenterology

Research and Practice
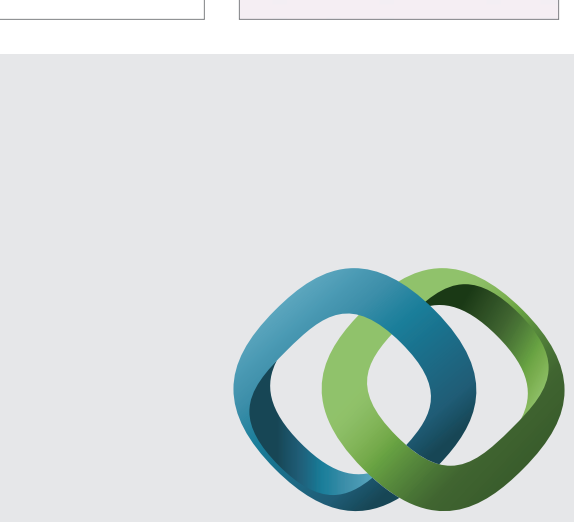

\section{Hindawi}

Submit your manuscripts at

http://www.hindawi.com
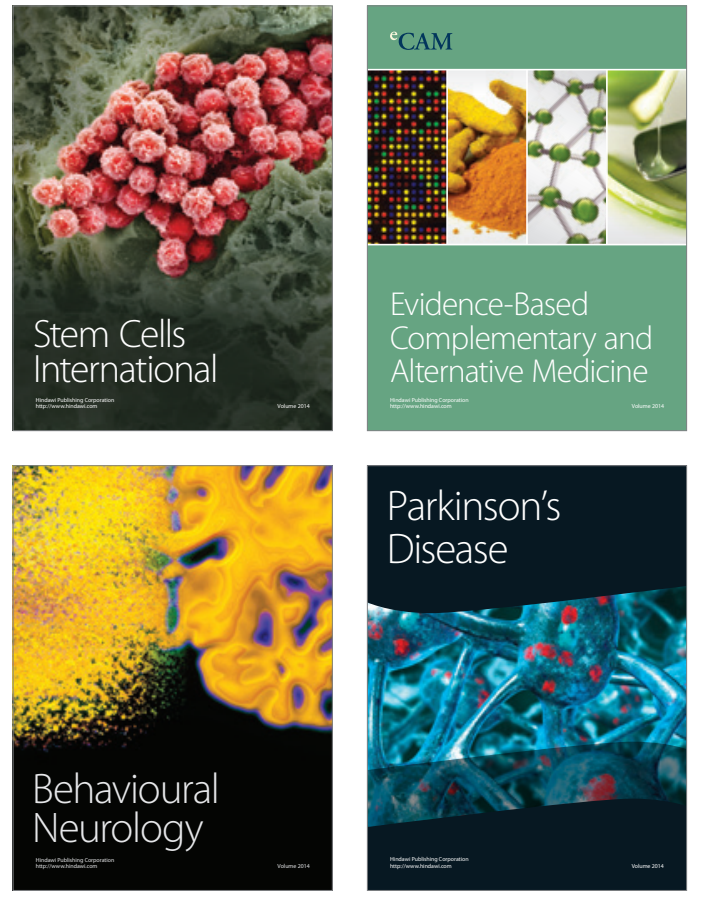
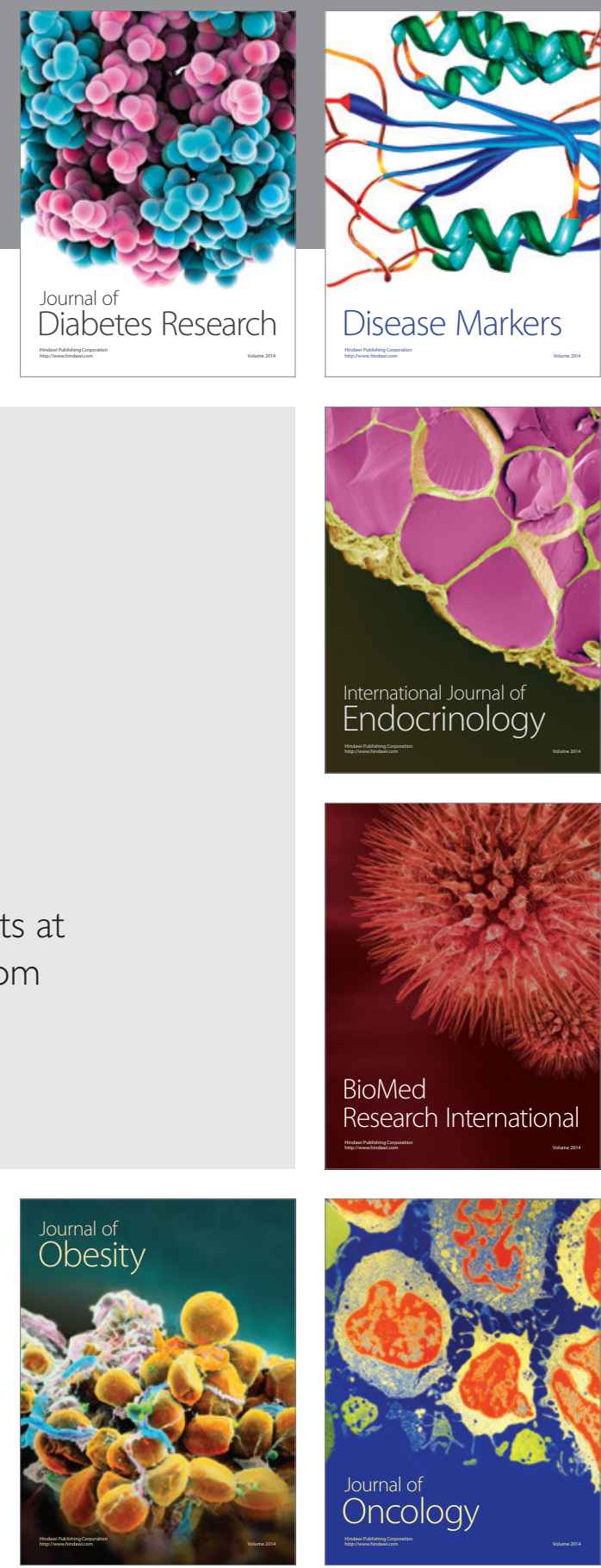

Disease Markers
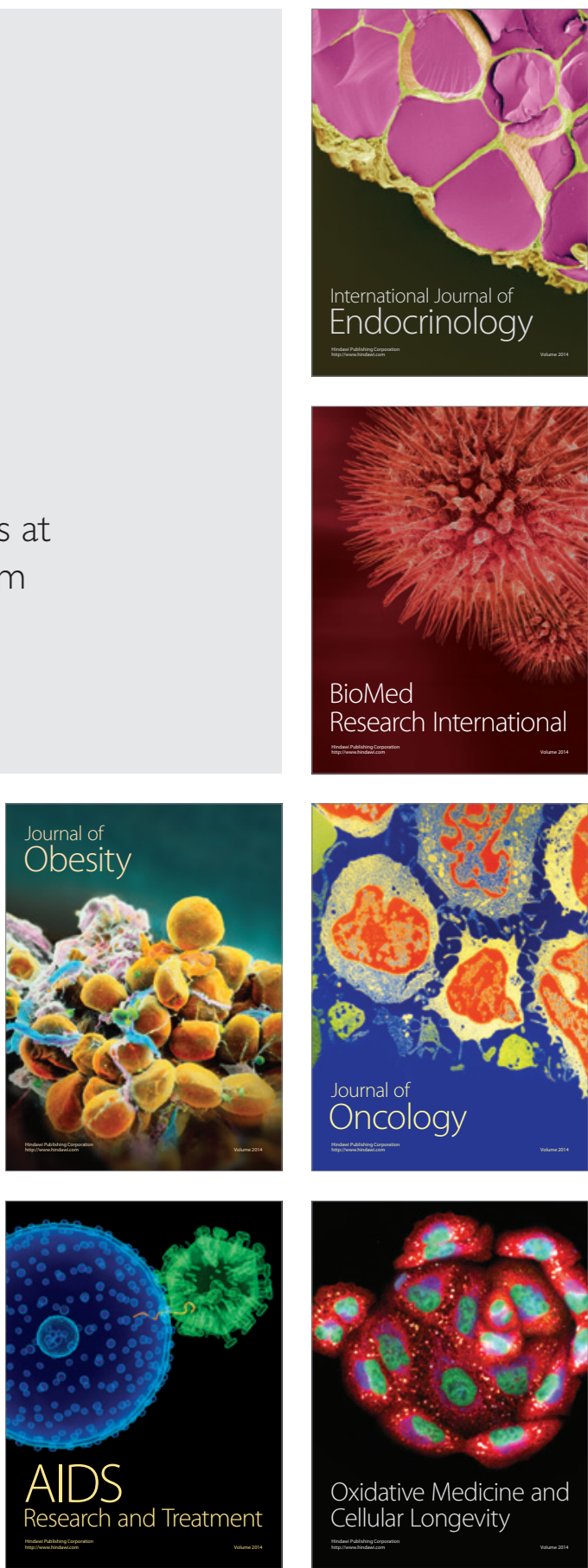